\title{
Frequency of polypharmacy and drug interactions in inpatients in the emergency department, Southwest of Iran
}

\author{
Mobina Tahmasebivand, ${ }^{1}$ Hassan Barzegari, ${ }^{2}$ Mandana Izadpanah ${ }^{3}$ \\ ${ }^{1}$ School of Pharmacy, Student Research Committee, Ahvaz. Jundishapur University of Medical Sciences, \\ Ahvaz; ${ }^{2}$ Department of Emergency Medicine, Golestan General Hospital, Ahvaz Jundishapur University of \\ Medical Sciences, Ahvaz; ${ }^{3}$ Department of Clinical Pharmacy, School of Pharmacy, Ahvaz, Jundishapur \\ University of Medical Sciences, Ahvaz, Iran
}

\begin{abstract}
This study aimed to evaluate the polypharmacy extent and the frequency and severity of drug interactions by evaluating inpatients in the emergency department. In this epidemiological-
\end{abstract}

Correspondence: Hassan Barzegari, Department of Emergency Medicine, Golestan General Hospital, Ahvaz Jundishapur University of Medical Sciences, Golestan Avenue, Ahvaz, Iran.

Tel.: +98.9143536843 - Tel./Fax: +98.6133743099.

E-mail: h_b1979@yahoo.com

Key words: Polypharmacy; drug interaction; emergency department.

Acknowledgments: This article is the result of a research project approved by the Ahvaz Jundishapur University of Medical Sciences. The authors would like to thank Dr. Fakher Rahim and Mr. Siavash Moulaie Birgani for their helpful advice and constructive comments.

Contributions: All authors contributed to the conception or design of the work; or the acquisition, analysis, or interpretation of data for the work, drafting the work or revising it critically for important intellectual content. All authors announce their final approval of the version to be published. Agreement to be accountable for all aspects of the work in ensuring that questions related to the accuracy or integrity of any part of the work are appropriately investigated and resolved.

Conflict of interest: The authors declare no conflict of interest.

Funding: This study was funded by Ahvaz Jundishapur University of Medical Sciences.

Ethics approval and consent to participate: The Ethics Committee of Ahvaz Jundishapur University of Medical Sciences approved this study (IR.AJUMS.REC.1397.706), and that the study is conformed with the Helsinki Declaration of 1964, as revised in 2013, concerning human and animal rights. All patients participating in this study signed a written informed consent form for participating in this study.

Availability of data and materials: This study's data will be available for all readers when completed.

Received for publication: 4 May 2020 .

Revision received: 24 November 2020.

Accepted for publication: 5 February 2021.

This work is licensed under a Creative Commons Attribution 4.0 License (by-nc 4.0).

${ }^{\circ}$ Copyright: the Author(s), 2021

Licensee PAGEPress, Italy

Emergency Care Journal 2021; 17:9082

doi:10.4081/ecj.2021.9082 descriptive study, data were collected retrospectively by reviewing medical records of 92 hospitalized patients in the emergency department with a stay over 48 hours. Out of the study population, $54.3 \%$ and $45.7 \%$ were respectively male and female, with a mean age of 59.09 . In terms of hospitalization, $27.2 \%$ and $16.3 \%$ were hospitalized due to heart problems and trauma, respectively and the mean length of hospitalization was 3.91 with a standard deviation of 2.57 days. The mean drug received was 8.48 , with a standard deviation of 4.48 . Of the patients, $81.5 \%$ received more than 5 drugs; in addition, the observed amounts of drug interactions of $\mathrm{A}, \mathrm{B}, \mathrm{C}, \mathrm{D}$, and $\mathrm{X}$ were $2.5 \%, 17 \%, 59.3 \%, 19.5 \%$, and $1.9 \%$, respectively. The drug interaction prevalence in inpatients in the emergency department was high. The presence of a pharmacist is necessary to identify drug interactions and reduce drug-therapy problems to provide quality services.

\section{Introduction}

One of the common methods in the treatment of patients is the use of medication, and its proper and rational prescription is one of the most important contributors to community health. ${ }^{1}$ Unreasonable administration and use of drugs are seen in many countries, including third world countries. ${ }^{2}$ Many doctors do not spend time for careful monitoring of all prescription and consumption drugs, and this inaccuracy in prescribing medications, especially for patients undergoing a multi-drug regimen, can lead to drug interactions. Patients with chronic disease resistant to treatment due to the prolonged course of the disease, which usually leads to the addition of other diseases to the primary problem, require multiple medications. ${ }^{3-5}$ The term polypharmacy, which has been common in recent years, has numerous definitions, but most researchers have considered using 5 or more drugs as polypharmacy. Polypharmacy can increase the risk of drug interactions, medication errors, side effects, hospitalizations, and treatment costs. ${ }^{6-8}$

One of the most important and common causes of patients referring to treatment centers is drug interactions, which is one of the most important medication errors. ${ }^{9}$ Drug interactions occur whenever the specific effect of a drug, which is prescribed for a patient, is changed by taking the second drug. Many of the side effects of prescription drugs are caused by a variety of drug interactions. Drug interactions themselves lead to a new disease in the patient, leading to more prescriptions of drugs to treat the patient. In addition to damaging different organs, it can increase patient costs, patient referrals, unnecessary para-clinical tests and consequently, the cost of treatment. The use of drugs that interact with each other will include a high percentage of the healthcare support budget of these patients, which may lead to a reduction in the number of services needed in this group. ${ }^{10}$

In the past decades, we have seen a link between the overcrowding of the emergency department and the quality of services 
provided. In addition to overcrowding, other factors such as stress, inadequate number of emergency medicine specialists, failure to communicate among the treatment team make the emergency department a place of occurrence of drug-treatment problems. In the emergency department, multiple drugs are prescribed concurrently, which are added to the patient's previous regimen without screening, thus exposing the patient to drug interactions. ${ }^{11,12}$

Since patients' health is a top priority for the health care system, drug interactions and their side effects should be avoided in order to improve their health more rapidly. It also doubles the importance of predicting drug interactions and how to treat them. ${ }^{10}$ Many drug interactions are predictable, and many of them can be prevented. Also, because in our country, it is not easy to measure the blood levels of drugs in patients, the importance of predicting drug interactions and how to deal with them is doubled. ${ }^{13}$ Therefore, we decided to evaluate the extent of polypharmacy and the prevalence and severity of drug interactions by evaluating these patients in the emergency department.

\section{Materials and Methods}

In this cross-sectional (epidemiological-descriptive) study, data from medical records of 92 hospitalized patients in the emergency department in Golestan Hospital, affiliated with the Ahvaz Jundishapur University of Medical Sciences, were collected retrospectively and randomly from 2018-03-21 to 2018-06-22.

Kardex forms of all patients referred to the Internal Emergency, Psychiatric, Cardiac, and Surgery departments during this period, and those patients who met the criteria were included in this study. Exclusion criteria were taking at least 2 drugs, staying at least 48 hours in the emergency department, incomplete records, and hospital stay for less than 48 hours. After collecting the data, the extracted data were inserted into the designed forms. These forms consisted of two parts, the first part consisting of demographic information, length of stay, hospitalization department, cause of hospitalization, underlying disease, and the second part included the name, dosage, and form of the received drug.

In this study, polypharmacy was considered concomitant use of 5 drugs or more, and drug interactions were determined by LexiComp software. In this software, drug interactions are classified into five classes of A, B, C, D, and X (Table1). After drug interactions were determined, the data were analyzed.

\section{Statistical analysis}

Values of quantitative variables (length of hospitalization, the number of prescription medications, and the number of drug interactions) are expressed as the mean \pm Standard Deviation (SD). Values of qualitative variables (gender, age groups, background disease, and cause disease) are presented as frequencies. The normality of data was assessed by the Kolmogorov-Smirnov test. Mann-Whitney test and Kruskal-Wallis test were used in order to compare mean values of quantitative variables in the levels of qualitative variables. Association between polypharmacy status and qualitative variables was analyzed using the chi-square test. Also, the association between quantitative variables was analyzed using the Spearman correlation coefficient test. A p-value of less than 0.05 was considered statistically significant. All data were analyzed with SPSS version 32 .

\section{Results}

Among the 92 patients included in this study, 42 patients $(45.7 \%)$ were male, and 50 patients $(54.3 \%)$ were female, with a mean age of 59.09 years. The total number of prescription drugs was 760 from 126 types, of which 409 were injectable drugs. The number of drugs received by patient was from 2 to 30 , with a mean of 8.48 and a standard deviation of 4.48 . A total of $81.5 \%$ of the study population had polypharmacy, of which $31.5 \%$ received more than 10 medications (Figure 1). The maximum frequency of individuals of hospitalization was in the internal emergency departments, and the minimum frequency of individuals of hospitalization was in the psychiatric department (Figure 2).

Table 1. Classification of drug interactions in the studied patients.

\begin{tabular}{ll}
$\begin{array}{ll}\text { Drug } \\
\text { interaction } \\
\text { class }\end{array}$ & Definition \\
A & There is no known drug interaction. \\
B & There is a drug interaction but it does not require clinical treatment. \\
\hline C & The drug interaction requiring treatment monitoring. \\
D & Drug interaction requiring dose adjustment. \\
\hline X & There is a drug interaction with an advised category for no concomitant use. \\
\hline
\end{tabular}

X There is a drug interaction with an advised category for no concomitant use.

\section{Received drugs}

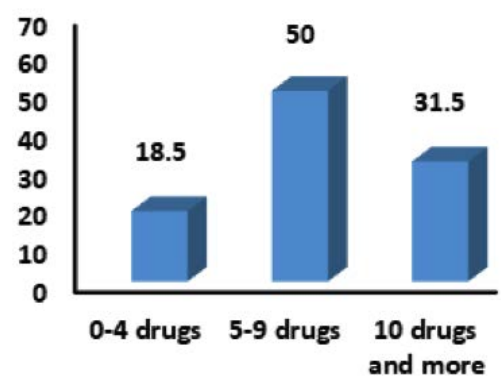

Figure 1. Frequency of receiving the drugs.

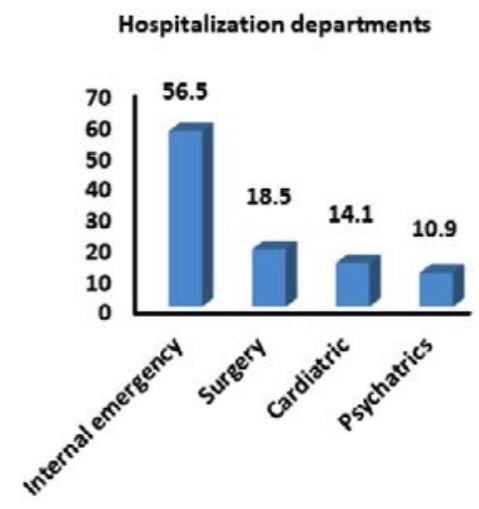

Figure 2. Frequency of individuals in the study departments. 
A total of 81 people (88\%) in the sample had at least one interaction. The number of drug interactions varied from 0 to 22 , with a mean of 5.52 and a standard deviation of 5.53 for each patient. Out of the patients, 9, 34, 81, 45, and 5 patients had interactions A, $\mathrm{B}, \mathrm{C}, \mathrm{D}$, and $\mathrm{X}$, respectively. The percentage and frequency of interactions are as follows (Table 2).

Most drug interactions have been between heparin and aspirin with 25 times administration. As Table 2 shows, the most frequent interactions were in the emergency departments.

In the terms of frequency, Class D/medium severity of drug interactions, frequencies of interactions of heparin and aspirin, phenytoin and ranitidine, and aspirin and clopidogrel were 25, 17, and 15 , respectively.

The most commonly prescribed drugs were pantoprazole (55 times) and atorvastatin (44 times). A list of the ten most commonly used drugs is shown in Table 3.

The number of drug interactions compared with demographic and clinical information of the patients is shown in Table 4.

Comparison between the number of drug interactions with demographic and clinical information of the patients are shown in Table 3. Results showed a positive significant relationship between drug interactions and age, length of hospitalization, and the number of drugs received (respectively $\mathrm{P}=0.022, \mathrm{P}=0.021$, and $\mathrm{P}<0.001)$. There was no statistically significant relationship between the number of drug interactions and gender $(\mathrm{P}>0.05)$.

Comparison between polypharmacy status and demographic and clinical information of the patients is shown in Table 5 .

As Table 5 shows, there was a significant statistical difference between mean values of number of prescription medications and number of drug interactions in patients with and without polypharmacy (respectively $\mathrm{P}<0.001$, and $\mathrm{P}<0.001$ ). Also, there was no significant statistical relationship between polypharmacy status and gender, age and length of hospitalization $(\mathrm{P}>0.05)$.

$\mathrm{X}$-class interactions were observed between the phenytoin drug with tenofovir, daclatasvir, and metronidazole ampoule and nimodipine drug, which was mostly seen in the surgical ward.

\section{Discussion}

When more than one type of medication is used in the treatment regimen of patients, the likelihood of drug interactions and subsequent complications of these interactions increases. ${ }^{14}$ The treatment team should identify and evaluate these interactions and prescribe an effective regimen for patients with the least complication and interactions..$^{15}$ In the present study, $81.5 \%$ of the subjects had polypharmacy and $31.5 \%$ received over 10 drugs.
Table 2. Percentage and frequency of drug interaction classes.

\begin{tabular}{lcc} 
Class of interactions & Frequency & Percentage \\
A & 9 & 2.5 \\
B & 61 & 17 \\
\hline C & 215 & 59.2 \\
D & 71 & 19.5 \\
\hline X & 7 & 1.9 \\
Total & 363 & 100 \\
\hline
\end{tabular}

Table 3. Ten most commonly used drugs by frequency and percentage.

\begin{tabular}{lcc}
\hline Drug & Frequency & Percentage \\
Pantoprazole & 55 & 7.2 \\
Atorvastatin & 44 & 5.8 \\
\hline Aspirin & 42 & 5.5 \\
Heparin & 39 & 5.1 \\
\hline Ceftriaxone & 31 & 4 \\
Ranitidine & 29 & 3.8 \\
\hline Regular Insulin & 24 & 3.2 \\
Dilantin & 24 & 3.2 \\
\hline Furosemide & 18 & 2.4 \\
Clopidogrel & 17 & 2.2 \\
\hline Total & 760 & 42.4
\end{tabular}

Table 4. Comparison between the number of drug interactions with demographic and clinical information of the patients $(\mathbf{n}=$ 92).

\begin{tabular}{|c|c|c|}
\hline Variable & Mean \pm SD & P-value \\
\hline $\begin{array}{l}\text { Gender } \\
\text { Male } \\
\text { Female }\end{array}$ & $\begin{array}{l}4.57 \pm(5.22) \\
6.32 \pm(5.59)\end{array}$ & $\mathrm{Z}=-1.75, \mathrm{P}^{*}=0.081$ \\
\hline Age & $r=0.24$ & $\mathrm{P}^{* *}=0.022$ \\
\hline Length of hospitalization & $r=0.241$ & $\mathrm{P} * *=0.021$ \\
\hline Number of prescription medications & $r=0.73$ & $\mathrm{P} * *<0.001$ \\
\hline
\end{tabular}

Table 5. Comparison between polypharmacy status and demographic and clinical information of the patients $(n=92)$.

$\begin{array}{lcc}\text { Variable Polypharmacy } & \text { P-value } \\ & \text { Nos } & (n=7)\end{array}$

Gender

Male

Female

Age $\quad 61.12 \pm 21.88$

Length of hospitalization

Number of prescription medications

Number of drug interactions

*: Chi-square test; **: Mann-Whitney U test.

\begin{tabular}{ccc}
$44(88 \%)$ & $6(12 \%)$ & $\chi^{2}=1.21, \mathrm{P}^{*}=0.27$ \\
$31(73.8 \%)$ & $11(26.2 \%)$ & $\mathrm{Z}=-1.389, \mathrm{P}^{* *}=0.165$ \\
$50.18 \pm 28.2$ & & \\
$4.05 \pm 2.77$ & $3.29 \pm 1.31$ & $\mathrm{Z}=-0.391, \mathrm{P}^{* *}=0.696$ \\
$9.60 \pm 4.22$ & $3.59 \pm 0.61$ & $\mathrm{Z}=-6.438, \mathrm{P}^{* *}<0.001$ \\
$6.56 \pm 5.60$ & $0.94 \pm 1.14$ & $\mathrm{Z}=-4.981, \mathrm{P}^{* *}<0.001$ \\
\hline
\end{tabular}


Polypharmacy can have many risks, including drug interactions for patients. Adverse effects on the consumer increased likelihood of inappropriate medication among drugs.

The study conducted by Perveen et al., who investigated the prevalence of polypharmacy in hospitalized patients in emergency departments in Pakistan, showed that prevalence of polypharmacy was $40 \%{ }^{16}$ Also in another study, its rate was $37.6 \%$, which has been significantly less than our study. ${ }^{17}$ In a study of polypharmacy in elders over 75 years old in London, conducted by Banerjee et al., polypharmacy rate was $45 \%{ }^{18}$ The incidence of polypharmacy has been significant in this number of patients admitted to the emergency department, and one of its causes may be the addition of many medications to the former patient's regimen without screening.

In a study conducted by Barot et al. the mean number of drugs received was 9.99 and the mean age of the subjects was 54.38 years. ${ }^{19}$ In our study, the mean number of drugs received, and the mean age of the subjects were 8.48 , and 59.09 , respectively. The total number of interactions in the present study was 363 , of which $2.5 \%, 17 \%, 59.22 \%, 19.5 \%$, and $1.9 \%$ were interferences $\mathrm{A}, \mathrm{B}, \mathrm{C}$, $\mathrm{D}$, and $\mathrm{X}$, respectively. In the study of patients admitted to the Sao Paulo emergency department, Okuno et al. identified 526 interferences. $^{20}$

In the study of medication prescriptions for patients discharged from the emergency department, conducted by Jawaro et al. (2015), out of the 429 known interactions, $15.6 \%, 60 \%, 22 \%$, and $1.6 \%$ were classes of B, C, D, and X, respectively. ${ }^{21}$ In the study in India in 2015, 1191 interactions were found, of which $29.89 \%$, $0.58 \%$, and $8.22 \%$ were minor, contraindicated, and severe, respectively, and average interactions for each patient was 7.63, but in the current study, it was $5.52 .{ }^{19}$ In a study conducted in the emergency department, Langdorf et al. found that $70 \%$ of patients had drug interactions, and $25 \%$ had severe interactions. ${ }^{22}$

The cause of the different prevalence of drug interactions in the different studies may be due to drug interference detection software or designing different studies. In this study, heart patients were more susceptible to interactions due to their higher mean age, multi-drug regimens, and underlying disease. According to the study conducted by Dookeeram et al., the drugs that cause the interaction were aspirin, lisinopril, and Clopidogrel, respectively. ${ }^{17}$ In our study, interaction-causing drugs were aspirin, heparin, and Clopidogrel, respectively.

In a study in 2018, Torkashvand et al. identified severe interactions, of which. Their most common were aspirin-clopidogrel (485 times), heparin-clopidogrel (329 times), and heparin-clopidogrel (199 times). These interactions need to be replaced by other medications by the treatment team if possible. ${ }^{15}$ Antiplatelet drugs are one of the most important drugs used in cardiac patients, of which the most widely used drugs in this category are aspirin. Combining aspirin with clopidogrel is the basis of treatment for the prevention of atherothrombotic events in cardiovascular patients, and existing guidelines recommend the use of these drugs in acute cardiac patients; each of these drugs affects platelet depletion in a different way. ${ }^{23} \mathrm{~A}$ number of clinical trials have shown better efficacy of concomitant use of aspirin and clopidogrel compared to aspirin alone. ${ }^{24}$ Medications should be taken with greater precision and monitoring at the same time.

According to the findings of this study, the greater the number of medications used, the greater the chance of drug interference. Other studies confirm this result, for example, the study on 11259 inpatients in a teaching hospital, conducted by Cruciol-Souza and Thomson, there was a linear relationship between drug number and drug interaction. ${ }^{25}$
The results of the present study also showed that the greater the number of drugs prescribed for patients, the greater the likelihood of drug interactions, which is consistent with the results of the study conducted by Nazari and Khanzadeh Moqhadam, and other studies. ${ }^{26-28}$

The findings of this study did not show any relationship between drug interactions and length of hospitalization. Previous studies have shown that increasing hospital stay increases the risk of drug interactions in patients. Murtaza et al.'s results also showed that people who stayed longer in the hospital were taking more medication and were at higher risk of interactions, consistent with our study. ${ }^{29}$

There was a significant relationship between drug interactions and age. As age increases, drug interactions increase. As the age increases, comorbidities become more common than ever, and chronic diseases are more common in the elderly. As a result, many patients, especially elderly people who take multiple medications, likely to develop drug interaction.

\section{Conclusions}

In emergency departments, all members of the treatment team, including nurses, should provide urgent care for critical patients and apply their clinical judgment of prescribed medications to prevent the possible drug interaction or reduction in the effectiveness of treatment. The presence of a clinical pharmacist will play a large role in such situations, including revisions to prescription to evaluate drug interactions and recommendations to prevent the occurrence of these interactions that will contribute to patient safety in the emergency department.

\section{References}

1. Moghadam Nia A, ZahedPasha Y, Mir Blooki M, et al. An analysis of prescription indices of Babol general practitioners prescriptions, 1999. J Babol Univ Med Sci 2000, 2:21-26.

2. Dolatabadi M, Jalili RH. Patterns of physicians'drug preh1ion in sabzevar iran (2008). J Sabzevar Uni Med Sci 2009;16:1616.

3. Ludgate J, Keating J, O'Dwyer R, et al. An improvement in cognitive function following polypharmacy reduction in a group of epileptic patients. Acta Neurol Scand 1985;71:44852.

4. Preskorn SH. Mania in a Case of Polypsychopharmacology: Pharmacodynamic and Pharmacokinetic Considerations Do You Believe in Magic? J Psychiatr Pract 2007;13:178-83.

5. Preskorn SH. Multiple medication use presenting as Parkinson's dementia complex: A message from Titanic. J Psychiatr Pract 2008;14:45-54.

6. Boparai MK, Korc-Grodzicki B. Prescribing for older adults. Mt Sinai J Med 2011;78:613-26.

7. Heuberger RA, Caudell K. Polypharmacy and nutritional status in older adults. Drugs Aging 2011, 28:315-23.

8. Kaufman DW, Kelly JP, Rosenberg L, et al. Recent patterns of medication use in the ambulatory adult population of the United States: the Slone survey. JAMA 2002, 287:337-44.

9. Khouri V, Semnani S, Roushandel G. Frequency distribution of drug interactions and some of related factors in prescriptions. 2006. Med J Tabriz Uni Med Sci 2006;27:29-32.

10. Esteghamat SS, Bastani F, Kazemi H, et al. Potential drug 
interactions in war-injured veterans with psychaitric disorders. 2011. Iran J War Public Health 2012;4:24-31.

11. McCarthy ML, Zeger SL, Ding R, et al. Crowding delays treatment and lengthens emergency department length of stay, even among high-acuity patients. Ann Emerg Med 2009;54:492503.

12. Gentile S, Vignally P, Durand A-C, et al. Nonurgent patients in the emergency department? A French formula to prevent misuse. BMC Health Serv Res 2010;10:66.

13. Rahimi B, Gharabaghi N, Hesari Z, et al. Prevalence of potential drug interactions in patients in the intensive care unit of Urmia Taleghani hospital. Urmia Med J 2013;24:133-45.

14. Roblek T, Deticek A, Leskovar B, et al, Clinical-pharmacist intervention reduces clinically relevant drug-drug interactions in patients with heart failure: a randomized, double-blind, controlled trial. Int J Cardiol 2016;203:647-652.

15. Torkashvand M, Esnaashari F, Mehrpoya M, et al. Evaluation of Potential Drug Interactions and Related Factors in Patients Admitted in Department of Cardiology of Farshchian Heart Hospital of Hamadan. J Clin Med 2018;25:105-111.

16. Perveen F, Khursheed M, Mujeeb R, et al. Incidence of Polypharmacy among emergency patients at a tertiary care Hospital in Karachi: an ignored paradigm for Quality Drug Therapy. Value Health 2015;18:A265.

17. Dookeeram D, Bidaisee S, Paul JF, et al. Polypharmacy and potential drug-drug interactions in emergency department patients in the Caribbean. Int J Clin Pharm 2017, 39:1119-27.

18. Banerjee A, Mbamalu D, Ebrahimi S, et al. The prevalence of polypharmacy in elderly attenders to an emergency department-a problem with a need for an effective solution. Int $\mathrm{J}$ Emerg Med 2011;4:22.

19. Barot PA, Malhotra SD, Patel VJ. Evaluation of Potential Drug-Drug Interactions in Patients of Emergency Medicine Department at a Tertiary Care Teaching Hospital: A
Prospective Study. Int J Sci Stud 2015;3:48-53.

20. Okuno MFP, Cintra RS, Vancini-Campanharo CR, et al. Drug interaction in the emergency service. Einstein (São Paulo) 2013;11:462-66.

21. Jawaro T, Bridgeman PJ, Mele J, et al. Descriptive study of drug-drug interactions attributed to prescriptions written upon discharge from the emergency department. Am J Emerg Med 2019;37:924-7.

22. Langdorf MI, Fox JC, Marwah RS, et al. Physician versus computer knowledge of potential drug interactions in the emergency department. Acad Emerg Med 2000;7:1321-9.

23. Pelliccia F, Rollini F, Marazzi G, et al. Drug-drug interactions between clopidogrel and novel cardiovascular drugs. Eur J Pharmacol 2015;765:332-6.

24. Manolis AS, Tzeis S, Andrikopoulos G, et al. Aspirin and clopidogrel: a sweeping combination in cardiology. Curr Med Chem Cardiovasc Hematol Agents 2005;3:203-19.

25. Cruciol-Souza JM, Thomson JC. Prevalence of potential drugdrug interactions and its associated factors in a Brazilian teaching hospital. J Pharm Pharm Sci 2006;9:427-33.

26. Abbasi Nazari M, Khanzadeh Moqhadam N. Evaluation of pharmacokinetic drug interactions in prescriptions of intensive care unit (ICU) in a teaching hospital. Iranian J Pharmac Res 2010:215-8.

27. De Almeida SM, Gama CS, Akamine N. Prevalence and classification of drug-drug interactions in intensive care patients. Einstein 2007;5:347-31.

28. Lima REF, Cassiani SHDB. Potential drug interactions in intensive care patients at a teaching hospital. Rev Lat Am Enfermagem 2009;17:222-7.

29. Murtaza G, Khan MYG, Azhar S, et al. Assessment of potential drug-drug interactions and its associated factors in the hospitalized cardiac patients. Saudi Pharmac J 2016;24:220-5. 